Glauber Dallanezi ${ }^{1}$

Eliana Aguiar Petri Nahas ${ }^{2}$

BeAtriz Funayama FreiRE ${ }^{3}$

JORGE NAHAS-NETO ${ }^{2}$

José EDUARDo CoRrente ${ }^{4}$

Gláucia Maria Ferreira da Silva Mazeto ${ }^{3}$

\title{
Qualidade de vida de mulheres com \\ baixa massa óssea na pós-menopausa
}

\author{
Quality of life of women with low bone mass in postmenopause
}

Artigo original

\begin{abstract}
Palavras-chave
Pós-menopausa Doenças ósseas metabólicas Osteoporose pós-menopausa

Questionários

Saúde da mulher

Qualidade de vida

Keywords

Postmenopause

Bone diseases, metabolic Osteoporosis, postmenpausal

Questionnaires

Women's health

Life quality
\end{abstract}

orrespondência:

Glaucia M. F. S. Mazeto

Departamento de Clinica Médica da Faculdade de Medicina de

Botucatu da UNESP

Rua Rubião Junior, s.n.

(EP: 18618-000 - Botucatu (SP), Bras

E-mail: gmazeto@fmb.unesp.br

Recebido

$19 / 10 / 2010$

Aceito com modificacōos

$31 / 10 / 2010$

\section{Resumo}

OBJETIVO: avaliar a qualidade de vida de pacientes com osteoporose e osteopenia, acompanhadas em ambulatórios especializados em osteoporose e climatério, comparando-as com pacientes com densidade mineral óssea (DMO) normal. MÉTODOS: estudo de série de casos transversal, observacional, que se propôs a analisar, por meio do questionário Medical Outcomes Study 36 Short-Form Health Survey (SF-36), a qualidade de vida de mulheres com osteopenia e osteoporose. Foram avaliadas 124 mulheres na pós-menopausa divididas em três grupos: 55 pacientes com diagnóstico densitométrico de osteoporose, 35 com o de osteopenia e 34 que apresentavam DMO normal. Os três grupos foram comparados com relação aos dados demográficos, características clínicas e de estilo de vida e aos diferentes domínios do SF-36. RESULTADOS: as

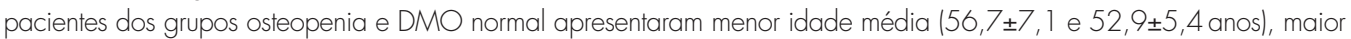

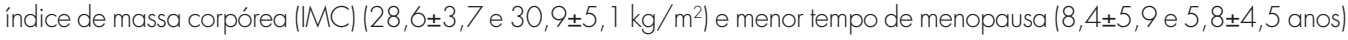
quando comparadas ao grupo osteoporose $161,8 \pm 10,1$ anos, IMC de 25,7 $5,3 \mathrm{~kg} / \mathrm{m}^{2}, 15,5 \pm 7,5$ anos, respectivamente; $\mathrm{p}<0,05)$. De acordo com o SF-36, não houve diferença significativa entre os grupos com relação aos domínios, à exceção do domínio vitalidade, que se mostrou superior no grupo osteoporose. Com relação à impressão pessoal sobre seu estado de saúde, das pacientes que o consideraram bom, um maior percentual pertencia ao grupo osteoporose, e entre aquelas que o consideraram ruim, um percentual menor pertencia ao grupo osteopenia. CONCLUSÃO: a qualidade de vida foi similar em mulheres com osteoporose e osteopenia, em relação às com DMO normal, à exceção do domínio vitalidade, que foi superior, paradoxalmente, nas pacientes com osteoporose.

\section{Abstract}

PURPOSE: to evaluate the quality of life of patients with osteoporosis and osteopenia followed-up at outpatient clinics specialized in osteoporosis and climacterium and to compare it to that of patients with normal bone mineral density (BMD). METHODS: cross-sectional case series, observational study, which intended to analyze the quality of life of women with osteopenia and osteoporosis by the Medical Outcomes Study 36 Short-Form Health Survey (SF-36) questionnaire. We evaluated 124 postmenopausal women divided into three groups: 55 patients with a densitometric diagnosis of osteoporosis, 35 with osteopenia and 34 who presented a normal BMD. The three groups were compared in terms of demographic data, clinical and life style characteristics and the different domains of SF-36. RESULTS: patients from the osteopenia and normal BMD groups presented lower age (56.7 \pm 7.1 and 52.9 \pm 5.4 years), greater body mass index (BMI) $\left(28.6 \pm 3.7\right.$ and $\left.30.9 \pm 5.1 \mathrm{~kg} / \mathrm{m}^{2}\right)$ and shorter time since menopause $(8.4 \pm 5.9$ and $5.8 \pm 4.5$ years $)$ than those from the osteoporosis group $\left(61.8 \pm 10.1\right.$ years, $\mathrm{BMl}$ of $25.7 \pm 5.3 \mathrm{~kg} / \mathrm{m}^{2}, 15.5 \pm 7.5$ years, respectively; $\left.p<0.05\right)$. According to SF-36, there was no significant difference between groups concerning the domains, except for the vitality domain, which was found to be superior in the osteoporosis group. Regarding the personal impression about their health, a greater percentage of the patients who considered it to be good was from the osteoporosis group and, of the patients who considered it bad, a smaller percentage belonged to the osteopenia group. CONCLUSION: the quality of life was similar in women with osteoporosis and osteopenia when compared to women with normal BMD, except for the vitality domain, which, paradoxically, was superior in patients with osteoporosis.
Faculdade de Medicina de Botucatu da Universidade Estadual Paulista "Júlio de Mesquita Filho" - UNESP - Botucatu (SP), Brasil. ' Professor da Faculdade de Educação Física de Barra Bonita - FAEFI - Barra Bonita (SP), Brasil.

2 Professores Assistentes e Doutores do Departamento de Ginecologia e Obstetrícia da Faculdade de Medicina de Botucatu da Universidade Estadual Paulista "Júlio de Mesquita Filho" - UNESP - Botucatu (SP), Brasil.

${ }_{3}^{3}$ Professora Assistente e Doutora do Departamento de Clínica Médica da Faculdade de Medicina de Botucatu da Universidade Estadual Paulista "Júlio de Mesquita Filho" - UNESP - Botucatu (SP), Brasil.

${ }_{4}^{4}$ Professor Assistente e Doutor do Departamento de Bioestatística do Instituto de Biociências da Universidade Estadual Paulista "Júlio de Mesquita Filho" - UNESP - Botucatu (SP), Brasil. 


\section{Introdução}

A qualidade de vida (QV) é um dos principais parâmetros pesquisados nos ensaios clínicos atuais. $\mathrm{Na}$ pesquisa de novas metodologias para tratamento e prevenção de doenças, surgiu a necessidade de padronizar sua avaliação. Para tanto, a ciência médica precisou definir o que entende por QV. A princípio, a QV pode ser definida como "o que cada um de nós considera como importante para viver bem" ${ }^{1}$, como, por exemplo, gozar de boa saúde e a possibilidade de usufruir momentos de lazer. No geral, pode-se dizer que se trata da sensação íntima de conforto, bem-estar ou felicidade no desempenho de funções físicas, intelectuais e psíquicas, dentro da realidade familiar, profissional e social ${ }^{1}$.

As doenças crônicas, as quais incidem particularmente em idosos, podem repercutir negativamente sobre a QV. Depressão/ansiedade, acidente vascular encefálico e osteoporose predispõem aos piores resultados ${ }^{2}$.

A osteoporose se constitui no segundo maior problema de assistência sanitária do mundo, seguida apenas pelas enfermidades cardiovasculares, segundo a Organização Mundial de Saúde (OMS) ${ }^{3}$, além de se constituir em um problema clínico e social de relevância, com sérias consequências para a saúde dos indivíduos ${ }^{4}$. Frequentemente os pacientes apresentam limitações para exercer normalmente suas atividades profissionais, sociais e de lazer, e apresentam uma afecção emocional importante ${ }^{5}$; esses fatores podem repercutir negativamente sobre a QV. Esta é considerada inferior em pacientes com osteoporose quando comparada à população geral, devido principalmente às fraturas decorrentes da baixa massa óssea, com diminuição da capacidade funcional ${ }^{6}$. O número e a severidade das fraturas podem determinar o grau de comprometimento da $\mathrm{QV}^{7}$. Além disso, os recursos humanos e financeiros utilizados no tratamento da osteoporose são significativos, com desequilíbrio na $\mathrm{QV}$ não só dos pacientes, como também de seus familiares ${ }^{8}$.

A avaliação da QV é realizada, na maioria das vezes, por questionários, podendo estes ser genéricos ou específicos ${ }^{9}$. Os questionários são multidimensionais, explorando diversos componentes da $\mathrm{QV}$, além de relevantes na avaliação das consequências das enfermidades crônicas. $\mathrm{O}$ questionário Medical Outcomes Study 36 Short-Form Health Survey (SF-36) ${ }^{10}$ é um instrumento genérico desenvolvido nos Estados Unidos para medir oito dos conceitos mais destacáveis relacionados à saúde. Aplica-se tanto à população geral como a pacientes específicos, sendo utilizado também em estudos de caráter descritivo ${ }^{6}$.

O objetivo do presente estudo foi avaliar a QV de mulheres com osteoporose e osteopenia, acompanhadas em um ambulatório especializado, comparando-as com pacientes com densidade mineral óssea (DMO) normal.

\section{Métodos}

Trata-se de um estudo de série de casos transversal, observacional e analítico que se propôs a analisar, por meio de questionário, a QV de mulheres com osteopenia e osteoporose. Este trabalho foi aprovado pelo Comitê de Ética em Pesquisa da Faculdade de Medicina de Botucatu da Universidade Estadual Paulista Júlio de Mesquita Filho (UNESP), em 6 de agosto de 2007, e todas as participantes concordaram e assinaram o Termo de Consentimento Livre e Esclarecido.

Foram avaliadas 124 mulheres no período pós-menopausa, acompanhadas em ambulatórios especializados no tratamento de osteoporose e climatério do Hospital das Clínicas (HC) da Faculdade de Medicina da UNESP, Botucatu (SP). As pacientes foram divididas em três grupos: 55 pacientes no grupo osteoporose, 35 pacientes no osteopenia e 34 pacientes no DMO normal. O número de pacientes estudadas baseou-se na estimativa de perda de $20 \%$ do total de casos com osteoporose (70 pacientes) e osteopenia ( 44 pacientes), acompanhadas nos ambulatórios ${ }^{11}$.

Os instrumentos utilizados foram o questionário SF-36, que já foi validado para a população brasileira ${ }^{12}$, e um breve questionário com dados gerais das pacientes, no qual constava sua impressão sobre o próprio estado de saúde (bom, regular ou ruim). Foram também avaliados os prontuários médicos para coleta dos seguintes dados: informações gerais, medidas antropométricas, medicamentos empregados no tratamento da baixa DMO, comorbidades (tais como hipertensão arterial, diabetes melito, osteoartrose, entre outras), diagnóstico de fraturas osteoporóticas, resultados dos exames de DMO de colo de fêmur (CF), coluna lombar (CL; intervalo de L2 a L4) e de triângulo de Ward (TW). As entrevistas foram realizadas após a consulta ambulatorial de rotina, sempre pelo mesmo profissional (pesquisador principal).

O SF-36 é um questionário multidimensional formado por 36 itens, englobados em oito domínios (componentes): capacidade funcional, aspectos físicos, dor, estado geral de saúde, vitalidade, aspectos sociais, aspectos emocionais e saúde mental. Os quatro primeiros domínios compõem o Sumário do Componente Físico (SCF), e os quatro últimos, o Sumário do Componente Mental (SCM). Para a obtenção dos escores, as questões devem ser pontuadas de acordo com normas pré-estabelecidas. Posteriormente, os valores das questões são transformados em escores, para cada um dos oito domínios. Assim, cada um desses componentes possui um escore, cuja pontuação varia de 0 a 100 , sendo 0 o pior e 100 o melhor estado de saúde. Cada domínio é analisado separadamente, não havendo um escore total. O SF-36 inclui, ainda, um item de avaliação das alterações de saúde ocorridas no período de um ano que não é pontuado (questão 2), mas é utilizado para comparação da saúde em geral ${ }^{12}$. 
As medidas antropométricas foram realizadas em balança com estadiômetro acoplado, marca Filizola ${ }^{\circledR}$, modelo LCD PL200. O índice de massa corpórea (IMC) foi calculado pela razão entre o peso $(\mathrm{kg})$ dividido pelo quadrado da altura, em metros $\left(\mathrm{m}^{2}\right)$, e classificado segundo os critérios da OMS, sendo considerados normais valores entre 18,5 e $25 \mathrm{~kg} / \mathrm{m}^{213}$.

As medidas de DMO foram realizadas na unidade de exames radiológicos do $\mathrm{HC}$ da Faculdade de Medicina de Botucatu pelo método DXA, e empregando-se o aparelho Soffus Lunar (LXRE). Os sítios considerados foram CF e CL (entre L2 e L4). Foram consideradas como portadoras de osteoporose, osteopenia ou DO normal as pacientes que apresentavam $\mathrm{DMO}$ com valores de T-escore iguais ou inferiores a $-2,5$, entre $-1,01$ e -2,49, e maior que $-1,0$ desvios padrão (DP), respectivamente. O diagnóstico densitométrico de osteoporose ou osteopenia baseou-se na observação de alteração em qualquer um dos sítios ósseos avaliados, mesmo na ausência de histórico de fratura osteoporótica ${ }^{3}$.

Os três grupos foram comparados quanto a dados gerais e clínico-laboratoriais, com a finalidade de caracterização das pacientes e quanto aos escores de cada domínio do SF-36.

Os parâmetros clínico-laboratoriais e terapêuticos das pacientes foram analisados utilizando-se estatística descritiva, com o cálculo de média e DP para as variáveis quantitativas, e frequência e porcentagem para as variáveis qualitativas para o geral e estratificado por grupo. Para a associação entre as variáveis qualitativas, utilizou-se o Teste Exato de Fisher, quando necessário. Os escores do SF-36 foram comparados, entre os três grupos, pela análise de variância (ANOVA), seguida pelo teste de comparações múltiplas de Tukey, utilizando-se idade, IMC e tempo de menopausa como variáveis de ajuste ${ }^{11}$. Os testes estatísticos foram bilaterais e o nível de significância adotado foi de $5 \%$. As análises foram realizadas utilizando-se Statistical Analyses System (SAS), versão 9.2, pelo Grupo de Apoio à
Pesquisa (GAP) da Faculdade de Medicina de Botucatu, que deu o atendimento metodológico e conduziu os procedimentos estatísticos.

\section{Resultados}

Neste estudo, a média da idade e do tempo de menopausa das mulheres do grupo com osteoporose foi superior aos dos demais grupos $(\mathrm{p}<0,05)$. A média do peso foi superior nos grupos DMO normal e osteopenia em relação ao grupo osteoporose $(\mathrm{p}<0,05)$. Quanto à estatura, o grupo osteopenia mostrou-se mais baixo em relação aos demais grupos $(\mathrm{p}<0,05)$. Porém, quando avaliado o IMC, os grupos DMO normal e osteopenia apresentaram médias significativamente superiores ao grupo osteoporose (Tabela 1).

Observou-se que um maior percentual de pacientes da raça branca era do grupo osteoporose $(43,8 \%)$, quando comparado aos demais grupos $(28,1 \%$, tanto no osteopenia quanto no DMO normal; $\mathrm{p}<0,05)$. Por outro lado, encontrou-se menor percentual de pacientes tabagistas no grupo osteopenia $(11,1 \%)$ em relação ao grupo osteoporose $(55,6 \%)$ e DMO normal $(33,3 \%$; $\mathrm{p}<0,05)$.

$\mathrm{O}$ tratamento utilizado pelas pacientes direcionado à abordagem da baixa massa óssea pode ser observado na Tabela 2. Considerando-se qualquer forma de tratamento, maiores percentuais foram observados no grupo osteoporose em relação ao osteopenia e, neste, em relação ao DMO normal ( $\mathrm{p}<0,0001)$. Das pacientes tratadas com dieta, alendronato e calcitonina, maiores percentuais eram do grupo osteoporose em relação aos grupos osteopenia e DMO normal $(\mathrm{p}<0,0001)$.

Entre as 124 pacientes, 68,3\% apresentaram comorbidades. As mais frequentes foram: hipertensão arterial $(39 \%)$, osteoartrose $(37,4 \%)$ e diabetes melito $(12,1 \%)$. O grupo osteoporose apresentou maiores percentuais de pacientes acometidas por outras doenças. Das pacientes com

Tabela 1 - Parâmetros clínico-laboratoriais* das 124 mulheres na pós-menopausa avaliadas, de acordo com o grupo (valores apresentados em média \pm desvio-padrão)

\begin{tabular}{|c|c|c|c|c|}
\hline Parâmetros clínico-Iaboratoriais & DMO normal $(n=34)$ & Osteopenia (n=35) & Osteoporose $(n=55)$ & Valor $p$ \\
\hline Idade (anos) & $52,9 \pm 5,4^{0}$ & $56,7 \pm 7,7^{0}$ & $61,8 \pm 10,1^{b}$ & $<0,0001$ \\
\hline Tempo de menopausa (anos) & $5,8 \pm 4,5^{a}$ & $8,4 \pm 5,9^{\circ}$ & $15,5 \pm 7,5^{b}$ & $<0,0001$ \\
\hline Peso (kg) & $76,9 \pm 11,7^{b}$ & $67,3 \pm 10,0^{b}$ & $62,4 \pm 13,8^{\circ}$ & $<0,0001$ \\
\hline IMC ( $\left(\mathrm{kg} / \mathrm{m}^{2}\right)$ & $30,9 \pm 5,1^{b}$ & $28,6 \pm 3,7^{b}$ & $25,7 \pm 5,3^{0}$ & $<0,0001$ \\
\hline DMO $\left(\mathrm{g} / \mathrm{cm}^{2}\right):$ CF & $0,9 \pm,{ }^{c}$ & $0,8 \pm 0,1^{b}$ & $0,6 \pm 0,1^{0}$ & $<0,0001$ \\
\hline DMO (desvio-padrão): CF & $0,2 \pm 0,9 c$ & $-1,5 \pm 1,7^{b}$ & $-2,8 \pm 0,8^{\circ}$ & $<0,0001$ \\
\hline DMO (desvio-padrão): CL. & $0,2 \pm 1,1^{c}$ & $-1,2 \pm 1,0^{b}$ & $-2,7 \pm 1,1^{0}$ & $<0,0001$ \\
\hline
\end{tabular}

*Média \pm desvio-padrão. Médias seguidas de mesma letra não diferem significativamente pelo teste de Tukey $(c>b>a)$, ao nível de $5 \%$ de probabilidade; $\mathrm{kg}=$ quilograma; $\mathrm{m}=$ metros; $\mathrm{kg} / \mathrm{m}^{2}=$ quilograma por metro ao quadrado; $\mathrm{g} / \mathrm{cm}^{2}=$ grama por centímetro ao quadrado; $\mathrm{IMC}=$ índice de massa corpórea; $\mathrm{DMO}=$ densidade mineral óssea; $\mathrm{CF}=$ colo de fêmur; $\mathrm{CL}=$ coluna lombar. 
Tabela 2 - Distribuição numérica e percentual das 124 mulheres na pós-menopausa avaliadas, de acordo com o grupo, em relação ao tratamento direcionado à osteoporose e à osteopenia

\begin{tabular}{|c|c|c|c|c|c|}
\hline \multirow{2}{*}{ Tratamento } & DMO normal & Osteopenia & Osteoporose & \multirow{2}{*}{ Total } & \multirow{2}{*}{ Valor $\mathrm{p}^{*}$} \\
\hline & $n(\%)$ & $n(\%)$ & $n(\%)$ & & \\
\hline Geral & $2(3,0)^{0}$ & $16(24,3)^{b}$ & $48(72,7)^{c}$ & 66 & $<0,0001$ \\
\hline Dieta & $10(15,9)^{\circ}$ & $17(26,9)^{0}$ & $36(57,2)^{b}$ & 63 & $<0,0001$ \\
\hline Terapia de reposição hormonal & $14(43,7)^{0}$ & $11(34,4)^{0}$ & $7(21,9)^{0}$ & 32 & 0,1765 \\
\hline Alendronato & $0(0,0)^{\circ}$ & $3(10,7)^{a}$ & $25(89,3)^{b}$ & 28 & $<0,0001$ \\
\hline Calcitonina & $0(0,0)^{0}$ & $0(0,00)^{0}$ & $23(100,0)^{b}$ & 23 & $<0,0001$ \\
\hline
\end{tabular}

*Teste de comparação de proporções por linha (c>b>a). Teste exato de Fisher.

Tabela 3 - Escore contínuo do Medical Outcomes Study 36 Short-Form Health Survey (SF-36)* das 124 mulheres avaliadas na pós-menopausa, de acordo com 0 grupo, em relação aos diferentes domínios (valores apresentados em média \pm desvio-padrão)

\begin{tabular}{|c|c|c|c|}
\hline Domínios & DMO normal $(n=34)$ & Osteopenia (n=35) & Osteoporose $(n=55)$ \\
\hline Capacidade funcional & $65,6 \pm 24,5^{\circ}$ & $72,4 \pm 21,3^{0}$ & $68,6 \pm 25,2^{0}$ \\
\hline Aspecto físico & $61,8 \pm 43,2^{\circ}$ & $71,3 \pm 36,0^{\circ}$ & $66,3 \pm 38,1^{0}$ \\
\hline Dor & $54,4 \pm 10,5^{0}$ & $56,5 \pm 9,2^{a}$ & $58,7 \pm 13,4^{0}$ \\
\hline Vitalidade & $49,8 \pm 24,7^{\circ}$ & $59,1 \pm 24,6^{\circ}$ & $65,8 \pm 27,3^{b}$ \\
\hline Aspectos sociais & $49,6 \pm 20,1^{\circ}$ & $40,4 \pm 20,2^{\circ}$ & $47,8 \pm 19,8^{\circ}$ \\
\hline Sumário do componente físico & $60,3 \pm 17,5^{0}$ & $66,7 \pm 14,5^{0}$ & $66,5 \pm 17,2^{0}$ \\
\hline Sumário do componente mental & $55,3 \pm 18,6^{\circ}$ & $55,6 \pm 23,1^{\circ}$ & $59,7 \pm 19,9^{a}$ \\
\hline Escore final & $58,4 \pm 15,5^{\circ}$ & $62,5 \pm 15,9^{\circ}$ & $64,0 \pm 16,6^{0}$ \\
\hline
\end{tabular}

*Média \pm desvio-padrão. Médias seguidas de mesma letra não diferem significativamente, ao nível de $5 \%$ de probabilidade, pelo teste de Tukey (b>a). DMO = densidade mineral óssea.

comorbidades em geral, $28,6 \%$ eram do grupo DMO normal, $25 \%$ do osteopenia e $46,4 \%$ do osteoporose $(\mathrm{p}=0,007)$. Daquelas com hipertensão arterial, $27,1 \%$ pertenciam ao grupo DMO normal, $25 \%$ ao grupo da osteopenia e $47,9 \%$ ao da osteoporose $(\mathrm{p}=0,03)$. Das pacientes com osteoartrose, $30,4 \%$ eram do grupo DMO normal, 19,6\% do osteopenia e $50 \%$ do osteoporose $(\mathrm{p}=0,007)$. O percentual de casos de diabetes melito não diferiu entre os três grupos $(33,3 \%$ do grupo DMO normal, $20 \%$ do osteopenia e 46,7\% do osteoporose, $\mathrm{p}=0,30)$. Nenhuma das pacientes apresentou o diagnóstico de fratura osteoporótica.

Quanto à impressão pessoal sobre seu estado de saúde, das pacientes que o consideraram bom, um maior percentual pertencia ao grupo osteoporose $(48,0 \%) \mathrm{em}$ relação aos grupos osteopenia e DMO normal (26\% em ambos; $\mathrm{p}<0,05)$. Das pacientes que o consideraram ruim, um percentual menor pertencia ao grupo osteopenia $(6,6 \%)$ em relação aos demais $(46,7 \%$ em ambos os grupos; $\mathrm{p}<0,05)$.

Com relação ao questionário SF-36, os grupos não diferiram de forma significante em nenhum dos domínios, com exceção do domínio vitalidade, que foi superior no grupo osteoporose (Tabela 3). As variáveis idade, IMC e tempo de menopausa não exerceram efeito sobre os resultados do SF-36, segundo análise estatística realizada.

\section{Discussão}

A osteoporose tem sido associada a uma pior $\mathrm{QV}^{14}$, que pode ser avaliada por meio de diferentes ferramentas. As mais utilizadas são os questionários, podendo ser genéricos ou doença-específicos. Embora os primeiros possam apresentar questões inespecíficas e os últimos possam ser mais direcionados, os questionários genéricos são muito utilizados por permitirem uma comparação de resultados entre diferentes morbidades ${ }^{15}$. Entre os questionários genéricos, o mais empregado é o SF- $36^{10}$, que se correlaciona com questionários direcionados à osteoporose $\mathrm{e}^{14}$.

No presente estudo, foi avaliada a QV, por meio do SF-36, em mulheres na pós-menopausa, com osteoporose e osteopenia, em relação a um grupo com DMO normal. Não foram observadas diferenças estatisticamente significantes entre os grupos na quase totalidade dos domínios, com exceção do domínio vitalidade, no qual, paradoxalmente, o grupo de mulheres com osteoporose apresentou maior pontuação. Esses resultados diferem dos de Maróti-Nagy e Paulik ${ }^{16}$ que, comparando mulheres húngaras na pós-menopausa, também portadoras de osteoporose, osteopenia ou DMO normal, observaram que as com osteoporose apresentavam pior $\mathrm{QV}$ que as demais ${ }^{16}$. Aranha et al. ${ }^{6}$, avaliando mulheres da cidade 
de Salamanca (Espanha) com osteoporose e média de idade semelhante à do presente estudo (65 anos), observaram pontuações inferiores nos domínios capacidade funcional, aspecto físico, dor, estado geral de saúde e vitalidade, e superiores nos domínios aspecto social, aspecto emocional e saúde mental ${ }^{6}$. Os autores concluíram que as pacientes com osteoporose apresentavam baixa qualidade de vida, sobretudo nas áreas físicas, quando comparada com valores da população espanhola em geral. Contudo, esse estudo não contou com Grupo Controle, e a comparação com a população em geral foi realizada com base em estudo anteriormente publicado. No Brasil, Lemos et al. ${ }^{17}$, estudando pacientes portadoras de osteoporose da cidade de Vitória (ES), com média de idade de 72 anos, observaram escores de qualidade de vida também inferiores aos do presente estudo, em todos os domínios, com exceção apenas dos domínios aspecto social e saúde mental, os quais apresentaram maiores pontuações ${ }^{17}$. De Oliveira Ferreira et al. ${ }^{14}$, avaliando pacientes com osteoporose em Campinas (SP), também relataram uma pior $\mathrm{QV}^{14}$. Considerando-se mulheres na pós-menopausa com faixas etárias semelhantes às do presente estudo, também na pós-menopausa, mas sem investigação da massa óssea, Navega e Oishi $(2007)^{18}$, estudando pacientes praticantes de atividade física da cidade de São Carlos (SP), encontraram um escore de qualidade de vida superior à do presente estudo em todos os domínios. A explicação provável para tal fato poderia residir na prática regular de atividade física daquelas pacientes ${ }^{18}$.

As causas para a discordância desse com outros estudos podem ser várias. Talvez a principal seja a não inclusão, por muitos autores, da presença de fraturas, muitas vezes subclínicas, como fator a influenciar na $\mathrm{QV}^{14}$. De fato, a osteoporose é uma doença silenciosa até a ocorrência de fraturas, principalmente as de quadril ${ }^{19}$ ou múltiplas de coluna ${ }^{15}$, que podem repercutir negativamente sobre a QV. Por outro lado, Lombardi et al. ${ }^{20}$, comparando pacientes com osteoporose com e sem fraturas de coluna com pacientes sem esse diagnóstico, não observaram diferenças entre os grupos nos escores do SF-36. Deve-se ressaltar que esses autores não especificaram a extensão das fraturas vertebrais ${ }^{20}$. Assim, para exercer uma real influência sobre a $Q V$, as fraturas precisariam ser clinicamente significantes, devendo ser considerados o tipo, a quantidade e a localização das mesmas. No presente estudo, embora não tenha sido avaliada especificamente a presença de fraturas, não foram encontradas evidências nos prontuários médicos que indicassem o diagnóstico destas. Talvez essa seja uma das explicações para os resultados contraintuitivos observados, com ausência de um pior escore no SF-36 no grupo osteoporose.
Outro fator a influenciar a QV seria o uso de terapia de reposição hormonal (TRH), a qual vem sendo associada à melhora da mesma, em mulheres na pós-menopausa. Porém, o benefício obtido parece ser custo-efetivo apenas para mulheres abaixo dos 59 anos de idade ${ }^{21}$, faixa etária inferior à do presente estudo. Estudos realizados no Brasil comparando mulheres na pós-menopausa, usuárias ou não de TRH, por meio do SF-36 $6^{22}$ ou outras ferramentas ${ }^{23}$, não têm encontrado diferenças na $\mathrm{QV}$, apesar de haver uma melhora em alguns aspectos relacionados à menopausa em si no grupo que recebeu TRH ${ }^{23}$. No presente estudo, não foram observadas diferenças entre os três grupos quanto ao uso de TRH ou à QV.

Existem ainda outros fatores que podem interferir na QV das pacientes, tais como idade, tempo de menopausa e IMC ${ }^{14}$. No presente estudo, devido à falta de homogeneidade entre os grupos com relação a esses parâmetros, e com o grupo osteoporose apresentando os dados mais desfavoráveis, poderia se questionar o quanto os mesmos influenciaram nos resultados referentes à QV. Assim, durante a análise estatística, foi realizado ajuste para essas variáveis, com a finalidade de se excluir possíveis interferentes. De qualquer forma, não foi encontrado um pior escore no grupo osteoporose.

Os achados referentes à QV foram reforçados pela impressão pessoal que as pacientes manifestaram sobre o seu estado de saúde, com um maior percentual do grupo osteoporose considerando-o bom, apesar do maior percentual de comorbidades apresentadas em relação aos demais grupos. Lemos et al. ${ }^{17}$ também referiram que a maioria de suas pacientes com osteoporose estava satisfeita com seu estado de saúde ${ }^{17}$.Considerando-se a faixa etária em si, Joia et al. (2007), avaliando idosos, sem o diagnóstico de baixa massa óssea, da mesma região desta pesquisa, também relataram que a maioria estava satisfeita com a vida ${ }^{24}$. Talvez a sensação de bem-estar esteja relacionada ao fato das pacientes estudadas estarem recebendo atenção de equipes multidisciplinares presentes nos ambulatórios onde eram realizadas as consultas de rotina. Ou seja, a sensação de estar sendo cuidada poderia influenciar na percepção pessoal sobre seu estado de saúde. Contudo, esta afirmação carece de comprovação científica.

No presente estudo, o questionário SF-36 permitiu a elaboração de um diagnóstico quanto à qualidade de vida em pacientes com baixa massa óssea, acompanhadas em ambulatórios especializados. Concluiu-se que a qualidade de vida foi semelhante em pacientes com osteoporose e osteopenia, em relação às de DMO normal, em quase todos os domínios. A exceção foi o domínio vitalidade, que foi superior, paradoxalmente, nas pacientes com osteoporose. As razões para esse achado permanecem a ser esclarecidas. 
1. Nobre MRC. Qualidade de vida. Arq Bras Cardiol. 1995;64(4): 299-300.

2. Lima $M G$, Barros $M B$, César $C L$, Goldbaum $M$, Carandina $L$, Ciconelli RM. Impact of chronic disease on quality of life among the elderly in the state of São Paulo, Brazil: a population-based study. Rev Panam Salud Publica. 2009;25(4):314-21.

3. Organización Mundial de la Salud (OMS). Informe preliminar y recomendaciones de una comisión de expertos de la Organización Mundial de la Salud sobre una estrategia global para la osteoporosis. Rev Esp Enfer Metab Óseas. 2000;9(2):78-83.

4. Pinto Neto AM, Soares A, Urbanetz AA, Souza ACA, Ferrari AEM, Amaral B, et al. Consenso Brasileiro de Osteoporose 2002. Rev Bras Reumatol. 2002;42(6):343-54.

5. Cook DJ, Guyatt GH, Adachi JD, Epstein RS, Juniper EF, Austin PA, et al. Development and validation of the mini-osteoporosis quality of life questionnaire (OQLQ) in osteoporosic women with back pain due to vertebral fractures. Osteoporosis Quality of Life Study Group. Osteoporos Int. 1999;10(3):207-13.

6. Aranha LLM, Mirón Canelo JA, Alonso Sardón M, Del Pino Montes J, Sáenz González MC. Qualidade de vida relacionada à saúde em espanholas com osteoporose. Rev Saúde Pública. 2006;40(2):298-303.

7. Rostom S, Allali F, Bennani L, Abouqal R, Haijaj-Hassouni $N$. The prevalence of vertebral fractures and health-related quality of life in postmenopausal women. Rheumatol Int. In press 2011 .

8. Kowalski SC, Sjenzfeld VL, Ferraz MB. Utilização de recursos e custos em osteoporose. Rev Ass Med Brasil. $2001 ; 47(4): 352-7$

9. Minayo MCS, Hartz ZMA. Qualidade de vida em saúde: um debate necessário. Ciênc Saúde Coletiva. 2000;5(1):7-31.

10. Ware JE Jr, Sherbourne CD. The MOS 36-item short-form health survey (SF-36). I. Conceptual framework and item selection. Med Care. 1992;30(6):473-83.

11. Zar JH. Biostatistical analysis. $4^{\mathrm{a}}$ ed. Englewood Cliffs: PrenticeHall; 1999

12. Ciconelli RM, Ferraz MB, Santos W, Meinão I, Quaresma MR. Tradução para a língua portuguesa e validação do questionário genérico de avaliação de qualidade de vida SF-36 (Brasil SF-36). Rev Bras Reumatol. 1999;39(3): 143-50.
13. Pastor PN, Makuc DM, Reuben C, Xia H. Chartbook on trends in the health of Americans. Hyattsville: National Center for Health Statistics; 2002

14. de Oliveira Ferreira N, Arthuso M, da Silva R, Pedro AO, Neto AM, Costa-Paiva L. Quality of life in women with postmenopausal osteoporosis: correlation between QUALEFFO 41 and SF-36. Maturitas. 2009;62(1):85-90.

15. Lips $P$, van Schoor NM. Quality of life in patients with osteoporosis. Osteoporos Int. 2005; 16(5):447-55.

16. Maróti-Nagy A, Paulik E. Factors influencing quality of life of Hungarian postmenopausal women screened by osteodensitometry. Int J Rehabil Res. $2011 ; 34(1): 73-8$.

17. Lemos MCD, Miyamoto ST, Valim V, Natour J. Qualidade de vida em pacientes com osteoporose: correlação entre OPAQ e SF-36. Rev Bras Reumatol. 2006;46(5):323-8.

18. Navega MT, Oishi J. Comparação da qualidade de vida relacionada à saúde entre mulheres na pós-menopausa praticantes de atividade física com e sem osteoporose. Rev Bras Reumatol. 2007;47(4):258-64.

19. Romagnoli E, Carnevale V, Nofroni I, D'Erasmo E, Paglia F, De Geronimo S, et al. Quality of life in ambulatory postmenopausal women: the impact of reduced bone mineral density and subclinical vertebral fractures. Osteoporos Int. 2004;15(12):975-80.

20. Lombardi I Jr, Oliveira LM, Monteiro CR, Confessor YQ, Barros TL, Natour J. Evaluation of physical capacity and quality of life in osteoporotic women. Osteoporos Int. 2004;15(1):80-5.

21. Salpeter SR, Buckley NS, Liu H, Salpeter EE. The cost-effectiveness of hormone therapy in younger and older postmenopausal women. Am J Med. 2009;122(1):42-52.e2.

22. Zahar SEV, Aldrighi JM, Pinto-Neto AM, Conde DM, Zahar LO, Russomano F. Qualidade de vida em usuárias e não usuárias de terapia de reposição hormonal. Rev Assoc Med Bras. 2005; 51 (3):133-8

23. Martins MAD, Nahas EAP, Nahas-Neto J, Uemura G, Buttros DAB, Traiman P. Qualidade de vida em mulheres na pós-menopausa, usuárias e não usuárias de terapia hormonal. Rev Bras Ginecol Obstet. 2009;31(4): 196-202.

24. Rolim LMC. Nível de atividade física associado ao estilo e satisfação com a vida da população idosa do Município de Botucatu-SP [dissertação]. Botucatu: Universidade Estadual Paulista; 2008. 